Sidonie Naulin: La blogosphère culinaire: Cartographie d'un espace d'évaluation amateur. In: Réseaux (183), 31-62 (2014).

France Telecom R\&D

The original publication is available at the publisher's web site: http://dx.doi.org/10.3917/res.183.0027

The MPIfG Journal Articles series features articles by MPIfG researchers and visiting scholars published in peer-reviewed journals. Max Planck Institute for the Study of Societies (MPIfG) Cologne | www.mpifg.de

\title{
LA BLOGOSPHÈRE CULINAIRE
}

Cartographie d'un espace d'évaluation amateur

Sidonie Naulin

\section{La Découverte | Réseaux}

\section{$2014 / 1-n^{\circ} 183$ \\ pages 31 à 62}

\section{ISSN 0751-7971}

Article disponible en ligne à l'adresse:

http://www.cairn.info/revue-reseaux-2014-1-page-31.htm

Pour citer cet article :

Naulin Sidonie, « La blogosphère culinaire » Cartographie d'un espace d'évaluation amateur, Réseaux, 2014/1 n¹83, p. 31-62. DOI : 10.3917/res.183.0029

Distribution électronique Cairn.info pour La Découverte.

(c) La Découverte. Tous droits réservés pour tous pays.

La reproduction ou représentation de cet article, notamment par photocopie, n'est autorisée que dans les limites des conditions générales d'utilisation du site ou, le cas échéant, des conditions générales de la licence souscrite par votre établissement. Toute autre reproduction ou représentation, en tout ou partie, sous quelque forme et de quelque manière que ce soit, est interdite sauf accord préalable et écrit de l'éditeur, en dehors des cas prévus par la législation en vigueur en France. II est précisé que son stockage dans une base de données est également interdit. 


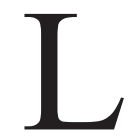

es blogueurs culinaires peuvent être définis comme des amateurs qui partagent une passion - la cuisine - sur un même support d'expression - Internet. Apparus à l'orée des années 2000, les blogs culinaires français ont connu une très forte croissance numérique au cours de la décennie suivante ${ }^{1}$. Leur développement s'inscrit à la fois dans le cadre du développement de la participation des amateurs sur Internet (Cardon, 2010 ; Flichy, 2010) et dans celui de la médiatisation croissante de la cuisine (prolifération des émissions télévisées, des magazines et des livres de cuisine). L'essentiel de l'activité des blogueurs culinaires consiste en la publication de recettes de cuisine. Toutefois, la réalisation d'évaluations concernant des produits alimentaires ou du matériel de cuisine fait aussi partie de leur répertoire de publications. Ces avis constituent, de même que les avis émis par des profanes sur les forums de discussion ou sur les sites de notation de produits (Beauvisage et al., 2013), un dispositif d'évaluation des biens gastronomiques concurrent des avis d'experts. L'émission d'avis par les blogueurs culinaires est d'ailleurs l'une des dimensions les plus médiatisées de leur activité : une grande partie des articles de presse consacrés aux blogueurs culinaires évoque en effet leur pouvoir " prescripteur », c'est-à-dire leur capacité à influencer les pratiques culinaires et les actes de consommation de leurs lecteurs. L'une des preuves les plus patentes de ce rôle de prescripteur peut être trouvée dans l'intérêt que les agences de communication, les marques de l'agroalimentaire et les fabricants de matériel de cuisine portent aux blogueurs qui sont sollicités pour évaluer leurs produits. Dans le blogging comme dans la presse culinaire (Naulin, 2012), l'évaluation n'est donc jamais très éloignée de l'information et de la promotion.

Dans cet article, nous cherchons à mettre au jour le(s) profil(s) de ces amateurs qui décident de produire des évaluations sur Internet dans les espaces de publication personnels que sont les blogs. À qui les internautes qui suivent les prescriptions des blogueurs font-ils confiance ? Il s'agit tout à la fois de cartographier l'espace de la blogosphère culinaire, mais aussi de parvenir à identi-

1. Au moment de notre enquête en mars 2011, Stéphane Gigandet, auteur du site Internet http:// recettes.de/cuisine, recensait 3268 blogs culinaires français actifs. 
fier les principes de hiérarchisation à l'œuvre en son sein. À la différence de la plupart des travaux académiques quantitatifs portant sur la notoriété des utilisateurs de plates-formes d'autopublication en ligne (Agarwal, Liu et Tang, 2008 ; Cardon, Fouetillou, Lerondeau et Prieur, 2011 ; Stoica, Couronné et Beuscart, 2010), nous ne partons pas des contenus mis en ligne, ni des liens entre usagers pour mettre au jour des hiérarchies, mais des pratiques et des propriétés des usagers eux-mêmes. Nous nous intéressons donc plus aux propriétés des individus qu'à celles des contenus qu'ils produisent.

Notre étude du monde des blogueurs culinaires se fonde sur des entretiens réalisés avec des blogueurs de cuisine ${ }^{2}(\mathrm{~N}=15)$, avec des évaluateurs professionnels que sont les journalistes culinaires $(\mathrm{N}=20)$ et avec des représentants de marques s'adressant aux blogueurs. Elle se base aussi sur une enquête quantitative menée en mars 2011 auprès de 1387 blogueurs culinaires français (621 réponses), ainsi que sur l'observation de deux événements où se rencontrent blogueurs et partenaires commerciaux (le Salon du Blog culinaire de Soissons en 2010 et le Kitchen Lab à Paris en 2012).

Dans une première partie, nous analysons le lien entre blogs culinaires, pratiques d'évaluation et marchandisation de l'évaluation. L'existence d'une évaluation "marchandisée », c'est-à-dire provoquée par les marques, permet d'objectiver une partie de l'activité évaluative des blogueurs. Dans une seconde partie, nous explorons les caractéristiques des blogueurs selon leur relation à la marchandisation de l'évaluation précédemment mise en évidence. Une typologie des blogueurs culinaires est alors établie.

2. Lorsque des extraits d'entretiens sont cités dans notre texte, nous indiquons, outre la qualité de blogueur de la personne qui parle, l'âge de son blog (en années) et le nombre de visiteurs uniques quotidiens du blog. Ces deux derniers éléments sont ceux qui discriminent le plus les blogueurs. 


\section{Qui sont les blogueurs culinaires? Quelques caractéristiques sociodémographiques}

- $94 \%$ des blogueurs culinaires de notre échantillon sont des blogueuses. La féminisation extrêmement forte de la blogosphère culinaire indique un positionnement des blogs davantage tourné vers la cuisine domestique quotidienne ${ }^{3}$ (essentiellement féminine) que vers la cuisine professionnelle (essentiellement masculine).

- La moitié des blogueurs a un âge compris entre 30 et 44 ans.

- $70 \%$ des blogueurs exercent une activité professionnelle.

- Bien qu'étant une activité amateur ne requérant pas de formation scolaire à l'entrée ${ }^{4}$, le blogging culinaire est le fait de personnes qui sont plus diplômées que la moyenne ${ }^{5}$. Cette caractéristique a déjà été mise en évidence pour d'autres groupes de blogueurs, mais elle peut paraître surprenante dans le cas des blogueurs qui parlent de cuisine. En effet, a priori, la cuisine n'est pas un sujet académique et elle semble être une thématique plus accessible que peuvent l'être d'autres sujets fréquents de blogs comme la politique ou les nouvelles technologies. Si ce ne sont pas les compétences culinaires qui sont discriminantes pour la création et l'entretien d'un blog de cuisine, ce sont plus probablement les compétences informatiques et littéraires, plus inégalement réparties au sein de la population.

- Bien qu'étant plus diplômés que la moyenne, les blogueurs culinaires sont en revanche assez représentatifs de la diversité des Français tant en matière de profession et catégorie socioprofessionnelle qu'en matière de revenu ou de localisation géographique.

\section{BLOGS, ÉVALUATION ET MARCHANDISATION DE L'ÉVALUATION}

\section{La place de l'évaluation dans les blogs culinaires}

Les blogueurs culinaires sont avant tout des amateurs de cuisine. Cette « passion ordinaire » (Bromberger, 1998) se traduit à la fois par une pratique

3. $41 \%$ des blogueurs utilisent spontanément l'expression « de tous les jours » pour qualifier la cuisine qu'ils présentent dans leur blog quand seulement $2 \%$ décrivent cette dernière comme « sophistiquée ».

4. À l'image des professionnels de l'évaluation culinaire que sont les journalistes gastronomiques, les blogueurs culinaires soulignent l'importance de l'apprentissage sur le tas des compétences mobilisées pour tenir un blog.

5. 52\% des blogueuses culinaires âgées de 35 à 44 ans ont un diplôme du supérieur contre seulement 16\% de l'ensemble des Françaises du même âge. 
intensive de la cuisine ${ }^{6}$ et par des investissements, notamment cognitifs ${ }^{7}$, permettant d'alimenter cette pratique. Le motif qui revient de manière récurrente pour expliquer la création du blog est celui de «partager sa passion » : $78 \%$ des blogueurs culinaires déclarent avoir créé leur blog dans cet objectif. Le Web 2.0 est un support particulièrement adapté à cette fonction. L'architecture technique des blogs permet l'instauration de « conversations » et elle rend également possible l'objectivation de l'assentiment des lecteurs au discours (commentaires, abonnement, compteurs de visites, etc.). La dimension relationnelle du blogging et les satisfactions qui lui sont affiliées sont donc primordiales pour les blogueurs culinaires. Généralement, la littérature sur la participation des amateurs sur Internet voit dans les blogs un « instrument d'expressivité personnelle » (Allard et Vandenberghe, 2003 ; Rouquette, 2008) et de " construction identitaire » (Fluckiger, 2006) permettant la validation de certaines facettes de la personnalité grâce à l'interactivité du support (Beaudoin et Velkowska, 1999 ; Cardon et Delaunay-Téterel, 2006). L'obtention de reconnaissance par le biais du partage de sa passion n'est toutefois pas le seul motif de création des blogs de cuisine. Les blogueurs indiquent très souvent que ce sont initialement les fonctions pratiques, voire utilitaires, du blog qui étaient recherchées : $54 \%$ d'entre eux disent ainsi avoir créé leur blog pour « conserver leurs recettes » ${ }^{8}$. Dans ce cas, le blog culinaire est envisagé comme un instrument d'archivage personnel, équivalent dématérialisé du traditionnel cahier de recettes familial ${ }^{9}$. La communication s'adresse alors moins (initialement à tout le moins) à un autrui généralisé qu'à des proches connus. Cela se traduit dans le type de rétribution symbolique qui est retiré du blog. Interrogés sur ce que leur apporte leur blog, les blogueurs classent les différents types de reconnaissance dans l'ordre suivant : en premier la satisfaction de soi (citée par $75 \%$ des blogueurs), puis la reconnaissance de son entourage (29\%) et enfin celle des autres blogueurs (27\%).

6. Les blogueurs cuisinent en moyenne 2 h 53 par jour en semaine et 3 h 19 par jour le week-end. Ces chiffres sont nettement supérieurs à ceux de l'ensemble de la population française : selon l'enquête Emploi du temps 2009-2010 de l'INSEE, les personnes " pratiquantes » (c'est-à-dire ayant cuisiné dans la journée) qui représentent $56 \%$ de la population, passent en moyenne $1 \mathrm{~h} 03$ à cuisiner pendant la semaine et $1 \mathrm{~h} 08$ le week-end.

7. Les blogueurs ont une consommation éditoriale particulièrement forte : la moitié d'entre eux possède plus de trente livres de cuisine et plus d'un blogueur sur dix (13\%) possède plus de cent livres de cuisine. Par ailleurs, $83 \%$ des blogueurs interrogés ont lu au moins un magazine culinaire au cours des deux mois précédant l'enquête et la moitié des blogueurs a lu plus de trois titres différents.

8. Plusieurs motifs pouvaient être simultanément mentionnés par les répondants.

9. La fonction de supplétif mémoriel tenue par la publication d'avis sur Internet a aussi été identifiée chez les critiques amateurs de livres sur Amazon (Pinch et Kesler, 2011). 
L'étude des motivations des blogueurs culinaires met donc en évidence que la publication d'évaluations de produits, d'ustensiles ou de biens gastronomiques à destination du grand public n'est pas la vocation première de la majorité des blogs de cuisine. Ces derniers sont d'abord tournés vers la publication de recettes de cuisine. Comme l'indique la figure 1, les recettes sont la thématique la plus systématiquement abordée dans les blogs culinaires. Cependant, nombreux sont les blogueurs qui parlent aussi de temps en temps de produits, d'ustensiles, de matériel de cuisine, de publications culinaires et d'événements gastronomiques au travers d'articles qui comportent une dimension évaluative. D'ailleurs, certaines recherches consacrées à des produits culinaires comportant les termes « avis » ou « choisir» conduisent principalement vers des blogs. Ainsi, lorsque l'on tape la requête « quel moule en silicone choisir » sur Google, cinq des dix premiers sites affichés comme résultats sont des blogs.

Figure 1. Thématiques abordées dans les blogs de cuisine

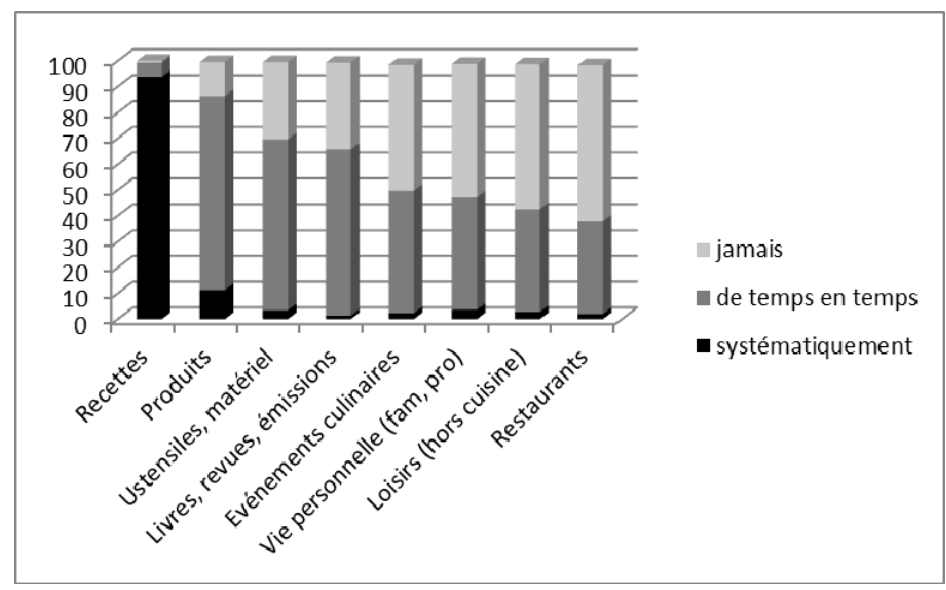

L'isolement de la dimension proprement évaluative des blogs de cuisine n'est pour autant pas évidente. S'il existe des articles spécifiquement dédiés à l'évaluation d'un produit où le blogueur réalise un test et donne son opinion ${ }^{10}$, l'évaluation est le plus souvent immergée dans un article plus général. Toute publication, même la plus descriptive, émet en effet indirectement une recommandation et peut être considérée comme prescriptive. Un blog qui propose

10. Voir un exemple en annexe 1. 
une recette à base de Nutella représente une forme indirecte de promotion (et donc de valorisation) de cette pâte à tartiner, celle-ci ayant été considérée comme la plus apte à assurer la réussite du plat présenté. L'évaluation, notamment dans son versant positif, est donc difficile à isoler de l'évocation, de l'information et de la recommandation qui imprègnent le contenu des blogs de cuisine. Plutôt que d'essayer de délimiter l'espace de l'évaluation profane au sein des blogs, nous attestons ici de son existence par la mise en évidence de ses conséquences les plus visibles : l'existence d'un intérêt d'acteurs économiques pour l'évaluation amateur véhiculée par les blogs.

\section{L'intérêt des marques pour l'évaluation amateur}

La manifestation la plus frappante du pouvoir prescripteur des évaluations produites par les blogueurs culinaires réside dans l'intérêt que les marques ${ }^{11}$ portent aux blogueurs. Nombreuses sont celles qui proposent divers types de collaboration aux blogueurs culinaires afin d'obtenir des articles sur leurs produits ou des recettes les mentionnant : envoi d'échantillons ou mise à disposition de produits, organisation de cours de cuisine, invitation de blogueurs à des voyages ou des déjeuners de presse, etc. Si les marques acceptent de payer pour que les blogueurs fassent, sous une forme ou sous une autre, la promotion de leurs produits, c'est qu'elles considèrent qu'ils ont la capacité, par l'émission de leur avis, d'influencer leurs lecteurs dans leurs actes d'achat.

Le « pouvoir» des évaluations émises par les blogueurs tient non seulement aux propriétés intrinsèques du média sur lequel s'expriment les blogueurs, mais aussi à la forme de confiance établie entre les blogueurs et leurs lecteurs. L'interactivité et l'instantanéité des réactions rendues possibles par les blogs et les réseaux sociaux permettent une circulation extrêmement rapide de l'information et elles donnent lieu à d'importants effets d'entraînement (modes, buzz, etc.) autour des informations produites par les blogueurs. Une blogueuse témoigne ainsi :

Les opérations [de communication] sont faites par les agences de relations presse des marques. Ça leur coûte moins cher que de la publicité, et du coup, ça marche bien. C'est relayé dans les forums, ça crée le forum. (Blogueuse, 3 ans, 800-1300 visiteurs)

11. Le mot marque désigne ici non seulement les marques de produits alimentaires, d'ustensiles de cuisine et d'art de vivre, mais aussi toutes les entreprises intéressées à la promotion que peuvent procurer les blogs (collectives de produits alimentaires, éditeurs de livres de cuisine, etc.). 
Le blogging est en effet une activité communautaire et c'est avec les autres blogueurs que s'instaurent les interactions les plus riches (commentaires réciproques, échange de lien entre les blogs, reprise et réinterprétation de recettes, demande de conseils, etc.), à la fois parce que les blogueurs souhaitent partager leur passion avec des amateurs qui leur ressemblent ${ }^{12}$ et en raison de l'existence de normes tacites de réciprocité des échanges :

« Chaque fois qu'on me met un commentaire, je vais répondre. Oui, je suis très attachée à ça aussi, c'est-à-dire que si on me met un commentaire, si [...] c'est quelqu'un qui a un blog, je vais aller moi en retour lui mettre un commentaire sur son blog, voilà, c'est de l'échange en fait.

Et ça c'est quoi ? C'est une règle?

Je pense que c'est une règle tacite. Enfin voilà, je pense que ça se fait comme ça. Je ne me suis pas posé la question en fait. On voit bien : si on pose un commentaire sur un blog qu'on ne connaissait pas, on voit bien que deux jours après on a un commentaire de cette personne qui, du coup, est venue visiter le blog et tout le monde fonctionne comme ça. » (Blogueuse, 1 an, 70 visiteurs)

La blogosphère culinaire est donc pour partie un univers autocentré (ce qui y est publié se destine prioritairement à ses membres) et mimétique (69\% des blogueurs culinaires déclarent trouver les idées des recettes pour leur blog... dans d'autres blogs). La sociabilité virtuelle développée entre les blogueurs tend, lorsqu'elle s'intensifie, à se transformer en sociabilité réelle puisque presque la moitié des blogueurs (49\%) ont déjà rencontré certains de leurs pairs. Cette sociabilité produit des effets d'émulation (« reprises» de recettes d'un blogueur à un autre), mais aussi d'incitation (à essayer de nouveaux produits ${ }^{13}$, de nouvelles techniques) et de dépassement de soi (s'améliorer tant au niveau de sa créativité que de sa technicité ${ }^{14}$ ).

12. Selon D. Cardon, G. Fouetillou et C. Roth (2011), l'importance des liens réciproques entre blogs est une caractéristique des blogosphères fortement cohésives centrées autour de thématiques féminines et domestiques.

13. Voici ce que dit une blogueuse :

Et vous cuisinez différemment maintenant que vous avez le blog?

Oui, je cuisine plus, et des produits différents. Par exemple des nouveaux produits qu'on voit sur d'autres blogs. On se dit que ça peut être sympa. Il y a plein de produits que je n'avais pas l'habitude de cuisiner et que j'ai faits en voyant d'autres recettes. Tout bête, le potimarron ou des trucs comme ça, je n'en faisais absolument jamais et à force d'en voir, de voir qu'on pouvait faire des trucs sympas... L'agar-agar ou ce genre de choses. (Blogueuse, 2 ans, 900 visiteurs)

14. La plupart des blogueurs rencontrés en entretien font en effet état d'une importante amélioration de leur compétence, notamment en cuisine, mais aussi en photographie et en écriture, 
L'intensité des échanges conversationnels sur les « forums » que sont les blogs permet ainsi aux marques de faire parler de leurs produits à un coût réduit (Mellet, 2009). Ces effets sont d'autant plus conséquents que la confiance accordée par les lecteurs aux informations émanant des blogueurs est relativement forte. Si, comme les journalistes culinaires, les blogueurs s'adressent à un public de passionnés, la confiance que leur octroie ce public ne repose pas sur les mêmes fondements. C'est justement parce que les blogueurs sont amateurs que leurs lecteurs suivent leurs recommandations. Selon une blogueuse :

Les marques nous sollicitent énormément parce que quand les blogueuses parlent d'un appareil, les ventes décollent. J'en suis la première « victime » : quand quelqu'un a parlé d'un appareil deux ou trois fois, je cours l'acheter. Il y a dans les blogs un ressenti, et donc une impression pour le lecteur d'être plus proche de la blogueuse que d'un magazine. (Blogueuse, 3 ans, 800-1300 visiteurs)

La proximité (il est possible d'interagir directement avec les blogueurs par le biais des commentaires), la moindre technicité (les blogueurs ne sont pas des professionnels de la cuisine ${ }^{15}$ ), la subjectivité (les blogueurs expriment leur ressenti personnel à la première personne) constituent les fondements de l'efficacité des évaluations émises par les blogueurs. C'est pourquoi en sus des évaluations que peuvent émettre spontanément les blogueurs, les marques sont enclines à leur faire produire des évaluations (positives) de leurs produits. Ce faisant, les formes mêmes de l'évaluation se trouvent modifiées.

\section{Les différentes formes d'interactions entre marques et blogueurs}

Les interactions entre les marques et les évaluateurs profanes prennent différentes formes et sont inégalement distribuées. Les blogueurs désignent sous le terme générique de " partenariats » ces transactions où une évaluation est produite en échange d'une contrepartie matérielle ou monétaire.

depuis la création de leur blog. Le statut de la « créativité » chez les blogueurs permet de mieux saisir ce phénomène. Initialement, seulement un tiers (32\%) des blogueurs créent leur blog avec pour objectif de développer leur créativité, mais a posteriori, plus de la moitié d'entre eux reconnaissent que le blog leur permet de développer leur créativité grâce à l'incitation à faire toujours mieux induite par la publication des recettes.

15. Voici comment une blogueuse raconte ce qui l'a séduite lorsqu'elle a découvert les blogs de cuisine :

Là, si tu veux, c'est des démos, la nana, elle n'a pas une cuisine professionnelle, elle a une cuisine comme toi donc quatre feux, un four, un congel', un fouet. Alors que sur les sites des chefs, la liste des ingrédients, tu fais : “OK, je n'en ai pas la moitié”, et des fois ils utilisent des termes... (Blogueuse, 2 ans, 1500 visiteurs) 
Contrairement à la publicité (présente sur $77 \%$ des blogs), dans le cas du partenariat, ce qui apparaît sur le blog n'est plus un message préfabriqué par la marque et identifié comme émanant d'elle, mais un message dont la teneur est co-construite, dans des proportions variables, par le blogueur et par la marque. Consécutivement, l'auteur du message devient plus flou que dans le cas de la publicité : s'agit-il d'une recommandation du blogueur lui-même ou d'un message déguisé de l'annonceur? Selon le poids accordé à chacun des deux partenaires, les modalités des partenariats diffèrent. Cinq types de partenariats, qui sont les plus fréquents, ont été retenus dans notre enquête. Ils sont classés par ordre croissant de subordination et de rémunération du blogueur : les échangesproduit où le blogueur reçoit gratuitement un produit à tester qu'il peut (ou parfois doit) évoquer ensuite dans son blog en donnant librement son avis ; les billets sponsorisés ${ }^{16}$ qui donnent lieu, en contrepartie de l'écriture de l'article, à une rémunération sous forme monétaire, l'évaluation du produit étant dans ce cas quasi obligatoirement positive ; et trois autres formes de travail rémunéré pour les marques qui requièrent un travail plus conséquent de la part des blogueurs (collaborations plus poussées avec les marques ${ }^{17}$, participation à un événement organisé par la marque comme un atelier de cuisine ou un salon culinaire, animation d'un événement) qui doivent là encore donner lieu à un compte rendu (élogieux) concernant les produits de la marque.

Tous les blogueurs ne sont pas concernés par les mêmes formes de relations avec les marques : un sur deux ( $55 \%)$ a déjà reçu des produits à tester, mais uniquement $8 \%$ ont déjà publié des billets sponsorisés. Le premier chiffre indique que les marques ne se focalisent pas uniquement sur les blogs les plus « influents », mais qu'elles peuvent chercher au contraire à atteindre la majeure partie de la blogosphère ${ }^{18}$. Le second indique que dès lors que la rémunération dépasse le simple envoi d'un produit, les marques sont beaucoup plus sélectives. La politique des entreprises qui s'intéressent aux blogueurs est donc double : d'un côté, les produits à tester sont envoyés le plus largement possible, tandis que de l'autre, les opérations rémunérées sont réservées à une élite. La réalisation de partenariats est fortement corrélée positivement à l'audience des blogs mesurée notamment en termes de nombre de visiteurs uniques quotidiens ${ }^{19}$ et de nombre

16. Un exemple de billet sponsorisé est présenté en annexe 2 .

17. Nécessitant la fourniture de recettes, l'animation d'une fan Page sur Facebook, etc.

18. Il faudrait bien sûr nuancer cette remarque selon le positionnement de la marque et la valeur unitaire de ses produits.

19. Les blogueurs qui ont la plus forte audience sont toutefois aussi ceux qui sont les plus nombreux à toujours refuser les partenariats. 
moyen de commentaires par billet. Elle est aussi positivement corrélée à l'investissement des blogueurs dans la promotion de leur blog sur Internet (possession d'une page Facebook pour le blog, édition d'une newsletter, référencement $\mathrm{du}$ blog, etc. $)^{20}$ et à leur originalité (appel à leur imagination pour trouver des recettes plutôt qu'à des sites de cuisine). La rémunération tirée du blog est quant à elle fonction du type et du nombre de partenariats réalisés.

\section{Motivations des blogueurs et conséquences sur l'émission de leur jugement}

Les apports économiques (sous forme monétaire, de cadeaux ou d'opportunités professionnelles) sont, dans les discours publics, régulièrement présentés comme un motif d'engagement dans la pratique du blog. La possession par certains blogueurs de cartes de visite professionnelles présentant leur blog indique bien qu'une partie d'entre eux est engagée dans une logique de promotion et de rentabilisation de l'activité. A posteriori, il semble toutefois que les apports d'ordre économique soient beaucoup moins importants pour les blogueurs que les apports symboliques. Seuls $2 \%$ des blogueurs déclarent parmi les satisfactions apportées par leur blog le fait qu'il leur rapporte de l'argent (il s'agit, parmi les dix items de réponse possibles, de l'item le moins fréquemment cité). La faiblesse de ce chiffre ne doit pas seulement être imputée à un phénomène de sous-déclaration. En effet, lorsque la question du montant rapporté par le blog est posée, seulement 3,5\% des blogueurs refusent de se prononcer et $35 \%$ déclarent que leur blog leur rapporte quelque chose. Les principaux apports matériels du blog sont en nature (produits, ustensiles, etc.) plutôt que monétaires et surtout les motivations matérielles sont, pour les blogueurs, moins importantes que les motivations symboliques.

L'absence de rémunération, équivalente à l'absence de partenariat, peut s'expliquer de deux manières : soit le blogueur n'a pas reçu de proposition de partenariat, soit il a reçu des propositions et les a déclinées. $14 \%$ des blogueurs culinaires disent ainsi refuser systématiquement les sollicitations des marques et $15 \%$ d'entre eux le font par principe (notamment pour conserver leur liberté éditoriale et la confiance de leur lectorat). S'ils ne sont pas majoritaires, les refus « par principe » sont néanmoins révélateurs d'une conception particulière de la blogosphère comme espace amateur et désintéressé où la passion,

20. La promotion de ses blogs par le biais de commentaires sur d'autres blogs a un effet plus nuancé : si elle favorise la probabilité de recevoir des produits à tester, elle réduit en revanche la probabilité de se voir proposer des collaborations plus poussées. 
la gratuité et l'amateurisme s'opposent au monde commercial des marques et des professionnels. D'autres motivations peuvent cependant aussi présider aux refus des partenariats. Il s'agit de l'absence d'intérêt pour le produit (qui intervient dans un refus de partenariat sur deux) et du désaccord quant aux conditions posées par la marque. Cela étant, la plupart des blogueurs acceptent les partenariats, ou à tout le moins une partie de ceux qui leur sont proposés, quand bien même la question de l'intervention des marques dans la production des évaluations est un sujet de débats au sein de la blogosphère culinaire.

Les blogueurs enthousiastes à l'égard des sollicitations dont ils sont l'objet ${ }^{21}$ le justifient de plusieurs manières : tout d'abord, cela leur permet de tester de nouveaux produits vers lesquels ils ne se seraient pas forcément tournés spontanément ou qu'ils n'auraient pas pu se payer ${ }^{22}$, ensuite, l'imposition d'une thématique (par exemple, cuisiner l'endive pour la marque Perle du Nord) leur permet de stimuler leur créativité. Enfin, pour ceux qui reçoivent une compensation monétaire, celle-ci leur permet de se défrayer des dépenses engendrées par le blog (paiement le cas échéant de la plate-forme hébergeant le blog, achat de l'appareil photo, etc.). Dans tous les cas, la production d'une évaluation n'est pas le motif premier d'acceptation des partenariats.

Lorsqu'il est question de l'influence de la transaction sur l'émission d'un jugement autonome, les blogueurs mobilisent un argumentaire et une rhétorique semblables à ceux rencontrés chez les journalistes culinaires qui sont eux aussi extrêmement sollicités par les marques. Les formes de partenariats proposées aux blogueurs sont en effet des extensions, dans le domaine de la blogosphère, de pratiques très courantes quoique moins visibles et plus réglementées, dans l'univers des médias traditionnels. Deux arguments sont avancés pour justifier du maintien de leur liberté éditoriale en dépit du partenariat : en premier lieu, les blogueurs disent sélectionner en amont uniquement les partenariats impliquant des marques ou des produits susceptibles de leur plaire (ce faisant, l'évaluation a lieu en deux temps : a priori au moment du choix d'évaluer au non le produit

21. Les blogueurs sont parfois aussi les initiateurs des partenariats : ils vantent alors auprès des marques l'impact positif d'une bonne critique de leur produit sur le blog afin de se faire offrir ce produit.

22. Ce sont des bons produits. J'ai reçu deux papillotes en silicone Mastrad, elles coûtaient 25 euros la papillote, je ne me les serais jamais achetées sinon. (Blogueuse, 2 ans, 100 visiteurs). J'aime bien parce qu'étant étudiante, moi, à un moment donné, ça m'a rendu service. Quand on m'a proposé le partenariat, je l'ai fait parce que recevoir des produits gratuitement, c'est le rêve. (Blogueuse, 2 ans, 900 visiteurs). 
puis au moment du test proprement dit) ; en second lieu, ils invoquent la possibilité de négocier avec la marque l'éventualité de ne pas parler d'un produit qui les aurait déçus lors du test ou d'euphémiser leur jugement si celui-ci est négatif $^{23}$. Voici comment une blogueuse raconte la construction de l'avis qu'elle a émis dans son blog à propos d'une préparation pour macarons vendue en grande surface. Après avoir accepté d'écrire un billet sponsorisé sur cette préparation, la blogueuse (qui maitrise par ailleurs parfaitement la technique traditionnelle des macarons) se rend compte que les macarons de la préparation sont « infaisables » et que c'est un « ratage ». Ne voulant pas tromper ses lecteurs, elle contacte la marque et lui propose soit de dire la vérité sur son blog, soit de ne pas écrire et d'arrêter le partenariat. Un compromis est finalement trouvé entre les intérêts de la marque et ceux de la blogueuse. Il s'agit, selon les termes de cette dernière, d'écrire le billet en " mentant par omission », c'est-à-dire en disant sur le blog que les macarons ne sont pas aussi bons que de vrais macarons maison, mais que la préparation peut servir en dépannage et faire l'affaire " lorsque l'on n'a que dix minutes et que sa belle-mère arrive ». La consultation de l'article rédigé révèle que l'euphémisation des critiques est finalement extrêmement forte sur le blog. En règle générale, les blogueurs complètent cette « euphémisation » publique des critiques par une sincérité « privée » : lorsqu'un lecteur leur demande par message privé ce qu'ils ont réellement pensé du produit, ils n'hésitent pas alors à exprimer leur ressenti réel.

Si elle n'est pas la vocation première des blogs culinaires, l'évaluation apparaît néanmoins comme une dimension importante de l'activité des blogueurs. Les formes qu'elle prend sont liées au niveau de "succès » des blogs. Pour mieux comprendre les liens entre ces deux phénomènes, nous étudions dans une seconde partie à partir de données quantitatives, les caractéristiques des blogueurs selon leur relation à la marchandisation de l'évaluation. Cela permet in fine de dresser une typologie des blogueurs culinaires.

\section{DISTRIBUTION DES PRATIQUES ET TYPOLOGIE DES BLOGUEURS}

\section{L'audience comme principe de hiérarchisation}

La question de l'évaluation produite par les blogueurs est inséparable de celle de l'efficacité sociale de cette évaluation, désignée comme prescription. Le

23. Les mêmes techniques sont utilisées par les critiques de restaurants invités par les attachés de presse (Naulin, 2010). 
pouvoir différentiel de prescription des blogueurs est considéré comme le principe fondamental de leur hiérarchisation. La compréhension du pouvoir de prescription des blogueurs intéresse aujourd'hui tant les sociologues que les acteurs eux-mêmes. C'est généralement le critère de l'audience, mesurée par le nombre de visiteurs uniques ${ }^{24}$, qui permet l'identification des blogueurs dont les avis comptent. Utilisé comme mesure du pouvoir de prescription des blogueurs, le nombre de visiteurs uniques est un indicateur central dans le monde des blogueurs. Ce critère est notamment utilisé par les partenaires commerciaux pour déterminer avec quel blogueur établir des partenariats ${ }^{25}$.

Figure 2. Répartition de l'audience ${ }^{26}$ totale des blogs entre les blogueurs

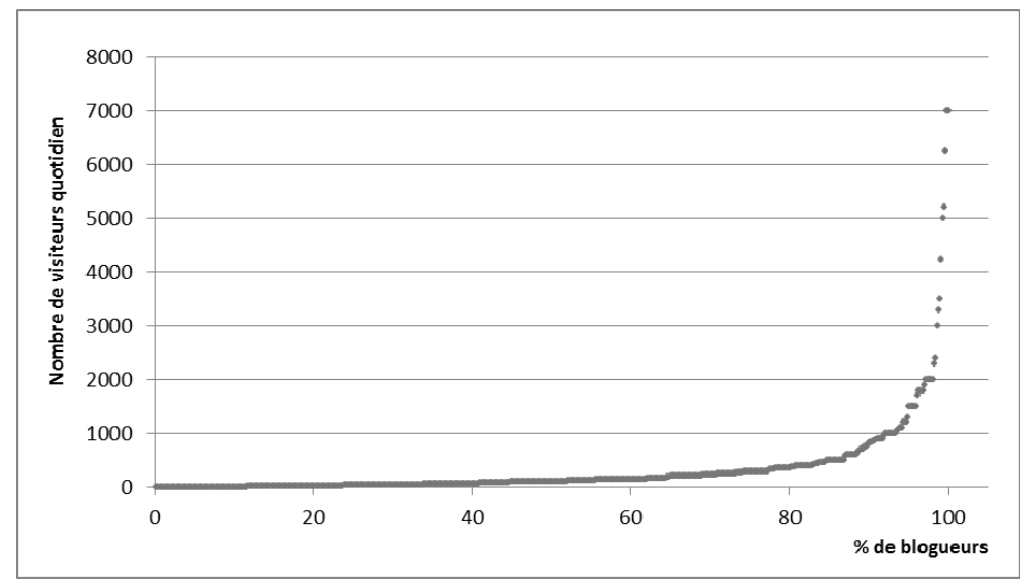

Lecture : $80 \%$ des blogueurs ont moins de 350 visiteurs quotidiens.

24. La question posée aux blogueurs concernant l'audience de leur blog était la suivante : «En moyenne, combien de visiteurs uniques avez-vous sur votre blog chaque jour / chaque mois?» La possibilité offerte au blogueur de donner le nombre de visiteurs quotidien ou mensuel est liée à l'hétérogénéité des chiffres mis à disposition des blogueurs par les plates-formes hébergeant les blogs ainsi qu'à l'écart important des ordres de grandeur de fréquentation selon les blogs. 25. Si les chiffres d'audience précis des blogueurs ne sont pas publics, il existe plusieurs classements de blogs et mesures (le blog rank par exemple) qui fournissent des informations globales. Les entreprises peuvent aussi passer par les plates-formes et demander directement aux blogueurs les plus visibles les chiffres de la fréquentation de leur blog mesurés par des outils standardisés. 26. En moyenne, un blog de cuisine reçoit 324 visiteurs uniques par jour, mais la moitié des blogs en ont moins de 100. Cette moyenne est tirée vers le haut par les quelques blogs à très forte fréquentation. 
Au sein de notre échantillon, comme dans d'autres univers en ligne (Cha et al., 2007 ; Beuscart et Couronné, 2009), la répartition de l'audience suit une loi de puissance (figure 2) : les $20 \%$ des blogueurs qui ont le plus de lecteurs réunissent $75 \%$ du lectorat total des blogs ${ }^{27}$. La blogosphère culinaire apparaît donc a priori comme un univers scindé entre une masse d'amateurs à l'audience réduite et une minorité de blogueurs disposant d'une audience forte et par extension d'un fort pouvoir de prescription.

L'audience est toutefois multiforme et sa mesure problématique. En effet, un nombre de visiteurs important peut traduire aussi bien une forte audience au sein de la blogosphère culinaire qu'à l'extérieur de celle-ci. Autrement dit, la reconnaissance dont bénéficie un blogueur peut être une reconnaissance de ses pairs et/ou une reconnaissance du grand public. Il est alors possible d'envisager non seulement une hétérogénéité de l'élite des blogueurs selon le type de reconnaissance dont ils bénéficient, mais aussi que les modalités de prescription varient selon le « marché » sur lequel est positionné le blog. Dans notre étude, trois indices sont utilisés pour hiérarchiser les blogueurs : le nombre de commentaires moyen par billet tout d'abord qui, dans la mesure où les blogueurs sont les principaux commentateurs des billets, est un indice indirect de l'intérêt que la blogosphère culinaire porte à un blog ; la mention du blog dans les médias ensuite ${ }^{28}$, qui est un indicateur de la reconnaissance " grand public » du blog, et enfin le nombre de visiteurs, qui synthétise ces deux premiers indices. Les trois mesures sont positivement corrélées (tableau 1).

Tableau 1. Corrélations de Pearson entre les indicateurs du succès des blogs

\begin{tabular}{|l|c|c|c|}
\cline { 2 - 4 } \multicolumn{1}{c|}{} & $\begin{array}{c}\text { Nombre de } \\
\text { visiteurs }\end{array}$ & $\begin{array}{c}\text { Nombre de } \\
\text { commentaires }\end{array}$ & $\begin{array}{c}\text { Nombre de mentions } \\
\text { dans les médias }\end{array}$ \\
\hline Nombre de visiteurs & 1 & $0,49 * *$ & $0,40^{* *}$ \\
\hline Nombre de commentaires & $0,49 * *$ & 1 & $0,15^{* *}$ \\
\hline Nombre de mentions dans les médias & $0,40 * *$ & $0,15^{* *}$ & 1 \\
\hline
\end{tabular}

** La corrélation est significative au seuil 0,01 (bilatéral).

27. Ces chiffres sont obtenus en faisant comme si chaque lecteur ne lisait qu'un seul blog. 28. Internet, presse régionale, radio ou télévision régionale, presse nationale, radio ou télévision nationale. Cette variable peut être transformée en échelle (nombre de types de médias différents dans lequel le blog a été cité). 


\section{Un espace structuré par l'intensité de la pratique et l'orientation intra- ou extrablogosphérique du succès des blogueurs}

À partir de cette mesure du succès d'audience des blogs, nous réalisons une analyse des correspondances multiples (ACM) afin de spécifier les propriétés des blogueurs. Deux types d'indicateurs sont retenus pour l'ACM : des indicateurs de pratiques (variables mesurant l'investissement des blogueurs dans l'activité de blogging) et des indicateurs de « succès » du blog (variables mesurant le succès d'audience et le succès commercial).

\section{L'analyse des correspondances multiples (ACM)}

L'analyse des correspondances multiples (ACM) est réalisée sur les 529 individus ayant au plus une non-réponse dans le questionnaire. 13 variables actives (ayant 52 modalités associées) sont retenues. Elles peuvent être regroupées en deux grandes familles :

1) Investissement du blogueur dans l'activité (indicateurs de pratique)

- Nombre d'heures passées à bloguer (5 modalités)

- Fréquence de publication des billets (6 modalités)

- Fréquence de publication des billets souhaitée à l'avenir (3 modalités)

- Fréquence de consultation des statistiques du blog (6 modalités)

- Participation à des forums de cuisine sur Internet (3 modalités)

- Niveau de difficulté des recettes (3 modalités).

2) Succès du blog

a) Succès d'audience

- Nombre de visiteurs uniques par jour (6 modalités)

- Nombre de commentaires par billet (4 modalités)

- Mention du blog dans les médias (3 modalités).

b) Succès commercial

- Type de partenariats réalisés (3 modalités)

- Nombre de produits testés au cours des deux derniers mois (4 modalités)

- Montant rapporté par le blog (3 modalités)

- Volonté de se professionnaliser (3 modalités) 
La figure 3 ci-dessous représente la projection des 529 blogueurs sur le plan formé par les axes 1 et 2 ainsi que les 30 modalités ayant le plus contribué à la formation des axes ${ }^{29}$.

\section{Figure 3. Analyse des correspondances multiples (individus et variables actives ${ }^{30}$ )}

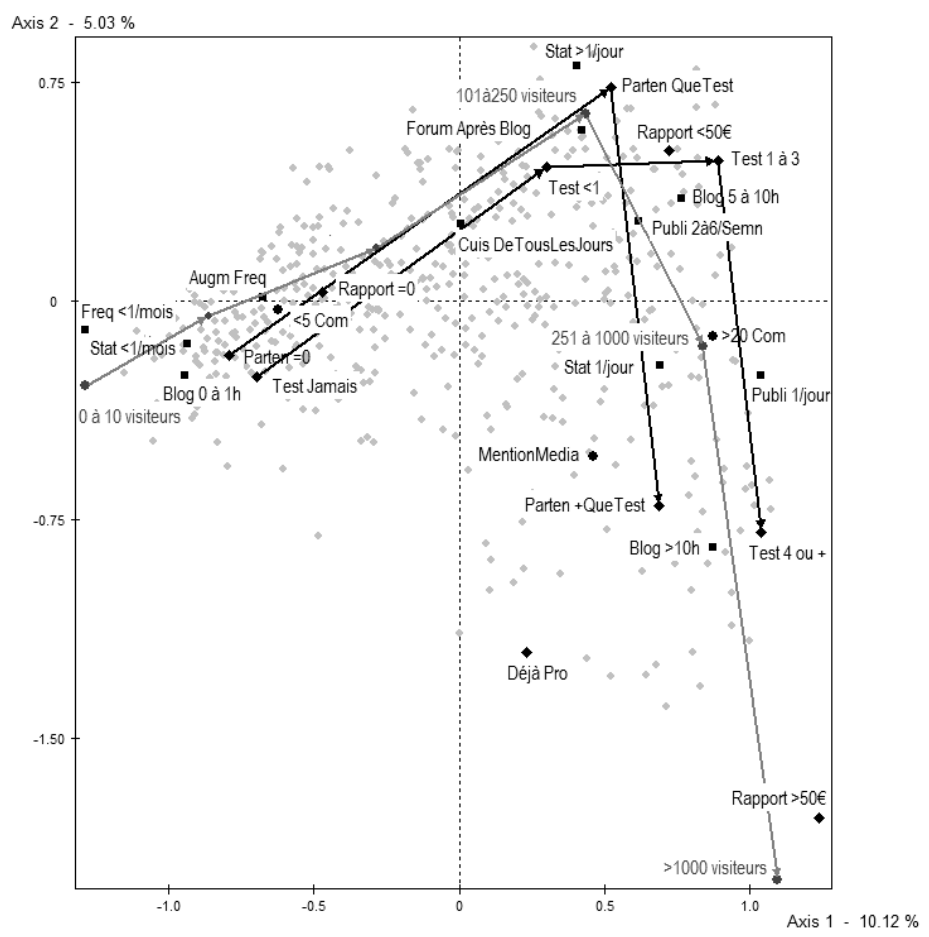

Légende :

Carrés : modalités des variables d'investissement du blogueur dans l'activité.

Ronds : modalités des variables de succès d'audience des blogs.

Losanges : modalités des variables de succès commercial des blogueurs.

29. N'ont été retenues que les modalités dont la contribution est supérieure à la contribution moyenne de l'ensemble des modalités pour l'axe.

30. L'index des variables et des modalités est donné en annexe 3. 
Le nuage des individus présente une forme en boomerang : plus on se déplace vers la droite du graphique, plus les individus sont dispersés de part et d'autre de l'axe horizontal (leur concentration est toutefois plus faible et leur dispersion plus forte dans le cadran sud-est que dans le cadran nord-est) avec un « espace inoccupé » entre le cadran sud-ouest et le cadran sud-est. La représentation graphique de l'analyse des correspondances multiples met bien en évidence cette forme boomerang lorsque sont dessinées les trajectoires des différentes variables dont les modalités contribuent fortement à la variance des axes. Par souci de clarté, seules les trajectoires de deux variables de succès commercial (types de partenariat et nombre de produits testés) et une variable de succès d'audience (nombre de visiteurs) ont été représentées.

Le premier axe, qui représente le meilleur ajustement unidimensionnel du nuage, restitue $10,12 \%$ de la variance totale du nuage. Les modalités qui contribuent le plus à la définition de l'axe 1 sont, à gauche de l'axe, celles qui témoignent d'une faible activité du blogueur et d'un faible succès du blog : jamais de partenariat, jamais de test, moins d'une publication de billet par mois, moins d'une heure par semaine passée à bloguer, moins de dix visiteurs uniques par jour, moins de cinq commentaires par billet, blog qui ne rapporte rien, volonté d'augmenter la fréquence de publication et statistiques du blog consultées moins d'une fois par mois. À l'opposé, les modalités qui contribuent le plus à l'axe 1 à droite de l'axe signalent une forte activité des blogueurs et un important succès du blog. Ce sont les modalités maximales des variables ordinales : au moins un produit testé au cours des deux derniers mois, blog qui rapporte quelque chose, plus de vingt commentaires par billet, publication d'au moins un billet par semaine, plus de 250 visiteurs uniques quotidiens, réalisation de partenariats, temps passé à bloguer compris entre cinq et dix heures par semaine, consultation des statistiques une fois par jour. Le premier axe dessine donc une opposition entre, d'un côté, les blogueurs faiblement investis dans leur pratique et à faible succès tant d'audience que commercial, et de l'autre, les blogueurs fortement investis dans leur pratique et à fort succès à la fois d'audience et commercial.

Le deuxième axe restitue $5,03 \%$ de la variance du nuage. L'ensemble des quinze modalités contribuant le plus à sa formation est situé dans le côté droit du graphique. Étant donné la forme du nuage, il apparaît que ce deuxième axe permet surtout d'opposer deux fractions au sein des blogueurs dont l'activité est moyenne à forte. Les modalités qui contribuent le plus à la définition de l'axe 2 sont, en dessous de l'origine, celles qui témoignent d'un très fort succès, notam- 
ment commercial, qui dépasse la stricte sphère de la blogosphère (mention du blog dans les médias et professionnalisation) : blog qui rapporte plus de $50 €$ par mois, plus de 1000 visiteurs uniques par mois, blogueur déjà professionnel de la gastronomie, réalisation de partenariats plus importants que des tests de produits, blog déjà mentionné dans les médias, au moins quatre tests de produits réalisés au cours des deux mois précédant l'enquête et plus de dix heures par semaine consacrées au blog. À l'opposé, les modalités qui contribuent le plus à la définition de l'axe 2 au-dessus de l'origine renvoient à une pratique et un succès réels, mais plus modérés et ne dépassant pas le cadre de la blogosphère. Ces modalités sont : partenariats limités aux tests de produits, nombre de visiteurs mensuels compris entre 101 et 250 , revenus engendrés par le blog inférieurs à $50 €$ par mois, participation à des forums de cuisine après la création du blog, moins de quatre tests de produits depuis deux mois (aucun ou un à trois), statistiques consultées plus d'une fois par jour et cuisine " de tous les jours » présentée sur le blog. Le deuxième axe dessine donc une opposition, d'autant plus importante que l'intensité de l'activité est plus forte, entre les blogueurs qui ont une activité moyenne et un succès limité à la blogosphère culinaire, et une minorité de blogueurs plus dispersée, au succès très fort qui dépasse le strict cadre de la blogosphère culinaire.

La projection de variables supplémentaires (liées à l'activité du blogueur sur Internet, à son rapport avec la cuisine et à ses caractéristiques sociodémographiques) permet de compléter l'interprétation des axes ${ }^{31}$. Les différents modes d'investissement personnel (dans le blog, dans la cuisine, dans la rentabilisation de l'activité) vont de pair selon le premier axe : plus les blogueurs sont situés à droite de l'axe, plus ils sont investis dans leur activité de blogueurs (abonnement à de nombreux blogs, rencontre de blogueurs, promotion de son blog en créant un compte Twitter), plus ils sont investis dans l'activité culinaire (nombre de livres de cuisine possédés, temps passé à cuisiner, participation à des concours de cuisine) et plus leur activité de blogueur est susceptible de leur apporter quelque chose et notamment des opportunités professionnelles. Il est notable que les variables sociodémographiques sont relativement peu pertinentes pour interpréter l'intensité de la pratique. Il s'agit là d'une différence forte avec les activités culturelles plus traditionnelles (arts, sports, loisirs, etc.) qui sont souvent fortement liées au capital culturel et économique des individus. L'indifférenciation des pratiques selon la profession

31. Seules les variables dont l'écart au point moyen est notable ou important sont retenues pour l'interprétation. 
et catégorie socioprofessionnelle (PCS) des individus est toutefois cohérente avec une récente étude sur les pratiques culturelles ${ }^{32}$ qui montre que, parmi neuf pratiques culturelles (lecture, bricolage, musique, sport, etc.), la cuisine (appréhendée par le fait de cuisiner des plats élaborés) est l'activité dont le taux de pratique présente pour les femmes la plus faible dispersion entre les PCS. Parmi les autres variables sociodémographiques, seuls le statut de l'activité (qui révèle une plus ou moins grande disponibilité temporelle) et l'âge sont liés à l'intensité de la pratique des blogueurs culinaires.

Le second axe peut s'interpréter comme un axe d'opposition qualitative entre les blogueurs. La qualité du blogueur est appréhendée à partir de deux types d'indicateurs : son niveau de compétences et la complexité de la cuisine qu'il propose. Toutes deux s'accroissent à mesure que l'on descend le long du graphique. L'une des questions de l'enquête portait sur le niveau de compétence des blogueurs dans quatre domaines : la cuisine, l'informatique, la photographie et la présentation des plats (esthétique). Il était demandé au blogueur de s'attribuer une note comprise entre 1 (mauvais) et 5 (professionnel) dans ces différents domaines. À partir des réponses à cette question, un indice synthétique de «niveau global» des blogueurs a été construit. Cet indice comprend quatre modalités : niveau faible, niveau moyen, niveau fort et niveau inégal (lorsque le blogueur est fort dans certains domaines et faible dans d'autres). Chaque blogueur se voit donc attribuer une seule évaluation de " niveau » mesurant sa compétence globale dans les différents domaines mobilisés par la tenue d'un blog. La représentation graphique de l'ACM montre que le niveau des blogueurs s'accroît à mesure que l'on descend le long du graphique ${ }^{33}$. De même, la cuisine proposée semble de plus en plus complexe : les blogueurs dont le succès dépasse la blogosphère culinaire lisent des magazines gastronomiques quasi professionnels (Thuriès) ou à la dimension artistique revendiquée (Fricote), ils possèdent beaucoup de livres de recettes et s'intéressent aux blogs consacrés au vin, tandis que les autres blogueurs lisent des magazines de recettes simples comme Guide Cuisine et s'inspirent des recettes publiées dans les autres blogs. L'opposition entre des blogs qui s'adressent à un cercle restreint de familiers et des blogs dont le succès dépasse la blogosphère est aussi marquée par les moyens de communication employés. La newsletter,

\section{Enquête ANR Dynegal (en cours).}

33. Il n'est toutefois pas possible de trancher sur le sens de la causalité : est-ce parce que les blogueurs sont meilleurs que leur blog rencontre un plus grand succès ou est-ce parce que leur blog rencontre un plus grand succès que les blogueurs se sentent meilleurs? 
destinée aux abonnés fidèles, est le propre des blogueurs du premier type. Les blogueurs de second type sont quant à eux cités dans les médias les plus sélectifs (presse nationale, radio et télévision). La «professionnalisation » de ces derniers est marquée par le fait qu'ils ont déjà exercé une activité liée à la cuisine et que leur blog est un moyen pour eux d'obtenir des opportunités professionnelles. Si les variables sociodémographiques sont peu discriminantes en ce qui concerne l'intensité de la pratique des blogueurs, elles le sont en revanche beaucoup plus dès lors qu'il s'agit de déterminer l'orientation locale ou globale du succès des blogs. Les hommes, les personnes les plus diplômées (au-delà de bac +4 ), les cadres et les artisans, commerçants et chefs d'entreprise, les personnes vivant dans des villes de plus de 100000 habitants et les personnes gagnant plus de 4000 euros mensuels sont ainsi clairement positionnés du côté des blogueurs tendant vers un succès qui dépasse le cadre de la blogosphère.

L'analyse des correspondances multiples met donc en évidence que le principal clivage entre les blogueurs porte sur l'intensité de leur activité. Aux blogueurs qui sont peu investis dans leur blog, dans la blogosphère culinaire et dans la pratique de la cuisine, s'opposent des blogueurs simultanément fortement investis dans ces différents domaines. Dans un second temps, les blogueurs fortement investis dans leur activité se distinguent entre eux selon leur orientation vers l'amateurisme et le succès au sein de la blogosphère ou leur orientation vers le succès à l'extérieur de la blogosphère et une éventuelle «professionnalisation».

\section{Quatre profils de blogueurs culinaires}

La réalisation d'une classification ascendante hiérarchique obtenue par regroupements successifs des blogueurs selon la proximité de leurs réponses aux questions utilisées comme variables actives fait apparaître une partition des blogueurs en quatre classes ${ }^{34}$ (figure 4 ). Les variables de succès commercial sont parmi les plus déterminantes dans la constitution des classes ${ }^{35}$. La partition permet donc de mettre au jour, de caractériser et de quantifier quatre types distincts de blogueurs culinaires selon leur rapport à la marchandisation et donc à l'évaluation.

34. Cette partition rend compte de $30 \%$ de la variance du nuage initial.

35. Voir annexe 4. 
Figure 4. Ellipses de concentration des 4 classes de la partition dans le plan de l'ACM

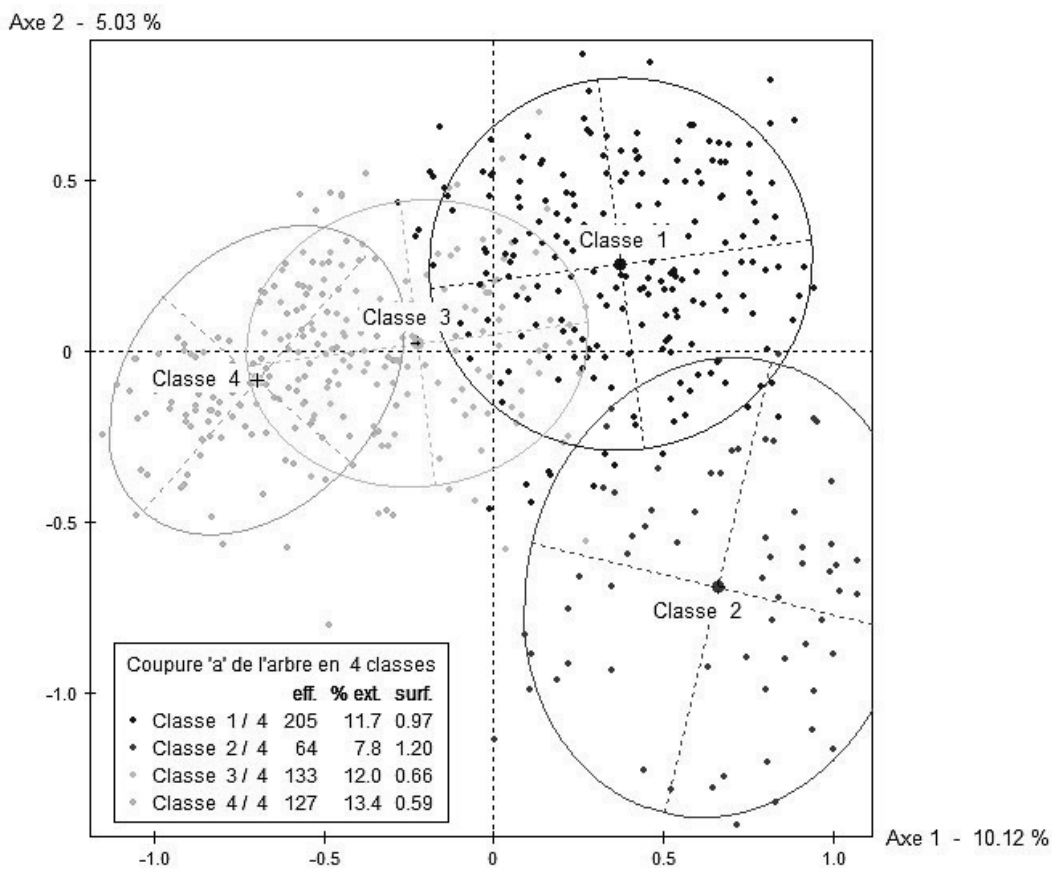

Figure 5. Dendrogramme partiel de la classification ascendante hiérarchique

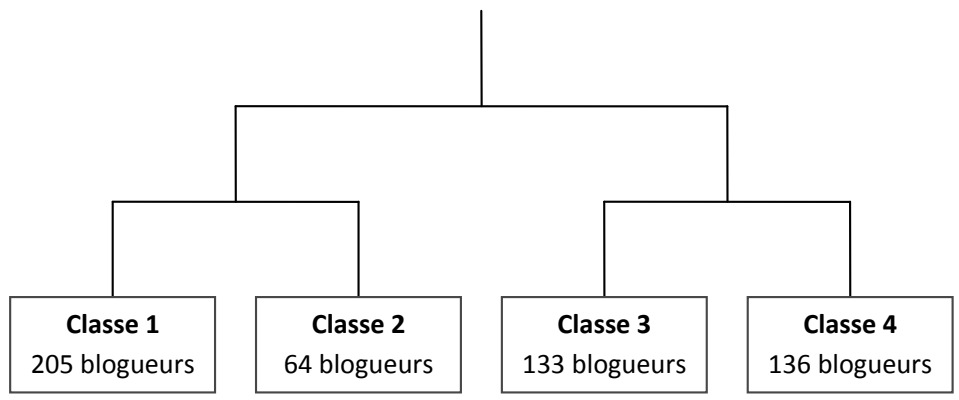

D'après le dendrogramme de la classification ascendante hiérarchique (figure 5), les classes 1 et 2 s'opposent aux classes 3 et 4. L'opposition la plus forte entre les classes, comme entre les individus, suit l'axe 1 de l'ACM, soit le niveau de pratique des blogueurs. D'un côté, les classes 3 et surtout 
4 regroupent les blogueurs à faible pratique et de l'autre, les classes 1 et 2 regroupent les blogueurs à forte pratique. L'opposition secondaire entre les classes 3 et 4 est aussi une opposition le long de l'axe 1 : les individus de la classe 4 sont ceux qui ont la pratique de blogging culinaire la plus faible. L'opposition secondaire entre les classes 1 et 2 est, en revanche, moins une opposition selon le niveau de pratique qu'une opposition selon l'orientation du blog vers la blogosphère culinaire ou vers l'extérieur : aux blogueurs de la classe 1 au niveau de pratique fort et tourné vers la blogosphère amateur s'opposent les blogueurs de la classe 2 au niveau de pratique très fort et tourné vers un succès externe. Les valeurs-tests permettent de sélectionner les modalités des variables les plus caractéristiques de chaque classe ${ }^{36}$ et ainsi de spécifier plus finement les quatre types de blogueurs mis en évidence.

Les classes 4 et 3, qui réunissent respectivement 24 et $25 \%$ des blogueurs, se caractérisent par les modalités minimales de succès commercial (aucun test, aucun partenariat, aucun apport monétaire du blog). Dans le cas de la classe 4, la déconnexion de l'espace marchand entourant les blogs va de pair avec un investissement minimal dans l'activité de blogueur (moins de un billet publié par mois, moins d'une heure par semaine de blogging, moins de 25 visiteurs par jour, moins de cinq commentaires par billet, etc.). Il s'agit donc de blogueurs marginaux. En revanche, les blogueurs de la classe 3 ont une activité de blogging un peu plus soutenue (publication une fois par semaine, 1 à 5 heures passées à bloguer par semaine, 26 à 100 visiteurs par jour, 6 à 10 commentaires par billet). Toutefois, si leur activité est non nulle, elle n'est en revanche pas suffisante pour permettre une insertion dans la sphère marchande. Cette situation d'amateurs non-prescripteurs semble leur convenir : ils souhaitent rester amateurs et à la différence des blogueurs marginaux, ils ne souhaitent pas augmenter leur fréquence de publication.

Les blogueurs de la classe 1, qui représentent $39 \%$ de l'ensemble des blogueurs, occupent le quart nord-est du graphique. Leur classe est celle qui regroupe le plus grand nombre d'individus. Elle se caractérise surtout par un succès commercial non nul, mais toutefois modéré du blog. À la différence des blogueurs des classes 3 et 4, les blogueurs de la classe 1 ont en effet entamé la marchandisation de leur blog (tests de produits, partenariats autres, apport monétaire du blog) et cela va avec une intensité du blogging plus forte.

36. C'est-à-dire les modalités qui sont significativement surreprésentées dans la classe par rapport à leur représentation dans la population globale. 
L'identification de cette classe permet de mettre au jour les seuils minimaux d'activité à partir desquels les blogueurs acquièrent une visibilité pour les marques (publier plus de 2 billets par semaine, passer plus de 5 heures par semaine à bloguer, avoir au moins 100 lecteurs quotidiens et plus de 10 commentaires par billet, etc.). S'ils aspirent à maintenir leur fréquence de publication de billets, les blogueurs de la classe 1 ne se caractérisent en revanche pas par leur volonté particulière de rester amateur. La classe 1 peut donc être vue comme une classe de transition entre les blogueurs qui souhaitent rester amateurs (classe 3 ) et ceux dont le succès dépasse la blogosphère (classe 2). Elle est celle des prescripteurs locaux.

La dernière classe, la classe 2 , occupe tout le cadran sud-ouest du graphique. Elle regroupe le plus faible effectif ( $12 \%$ de la population) et les individus y sont très dispersés. Cette classe se caractérise d'abord par un très fort succès commercial : la modalité la plus caractéristique de la classe est «blog qui rapporte plus de 50 euros mensuels ». Les blogueurs qui réalisent des partenariats plus poussés que les tests de produits et ceux qui testent beaucoup de produits (4 ou plus) y sont surreprésentés. Le fort succès commercial va de pair avec un fort succès d'audience (plus de 1000 visites quotidiennes, plus de 10 commentaires par billet, plus de 10 heures par semaine passées à bloguer, plus de deux billets publiés par semaine et consultation quotidienne des statistiques du blog). La classe 2 réunit donc l'élite des blogueurs dont le pouvoir de prescription dépasse le simple cadre de la blogosphère culinaire (mention du blog dans les médias, blogueurs déjà professionnels dans un domaine lié à la cuisine). Cette élite est minoritaire et très dispersée. Il n'existe donc pas un seul profil de blogueur à succès. Autrement dit, les blogueurs qui réussissent se ressemblent moins entre eux que les blogueurs qui ont une faible activité et une absence de succès commercial.

L'étude statistique de la population des blogueurs culinaires permet de mettre au jour la « structure chiasmatique » (Bourdieu, 1989) de leur espace social. Ce dernier est structuré dans un premier temps par une opposition entre blogueurs faiblement actifs et blogueurs très actifs et, dans un second temps, par une opposition entre pôle amateur et pôle commercial-professionnel. Ce résultat est congruent avec la typologie des blogs culinaires établie par D. Cardon, G. Fouetillou et C. Roth (2011) à partir de l'étude des liens entre les blogs de cuisine. Les auteurs mettent notamment en évidence deux trajectoires d'accession à la notoriété des blogs de cuisine, l'une conduisant à la reconnaissance par les pairs (passage d'un blog « journal intime » à un blog 
de cuisine spécialisé) et l'autre amenant à une reconnaissance plus universelle par une " professionnalisation » relative passant notamment par l'établissement de partenariats. Notre étude des propriétés des blogueurs montre que le rapport avec la production d'évaluations sollicitées par les marques est structurant pour différencier ces deux types de notoriété. Ce sont à la fois la quantité et la qualité (le type) de partenariat, et donc la forme de la recommandation qui changent, dès lors que le blog passe d'une audience locale spécialisée à une audience plus généraliste.

\section{Conclusion}

L'étude de la blogosphère culinaire par le prisme des propriétés des blogueurs permet de représenter leur espace, de mettre au jour les principes de leur hiérarchisation et de quantifier et qualifier les différents groupes qui la composent, notamment au regard de leurs activités d'évaluation.

La blogosphère culinaire apparaît structurée selon l'intensité de la pratique des blogueurs, leur succès d'audience et leur rapport aux marques. L'évaluation de produits et la marchandisation du blog sont rarement à l'origine du démarrage d'un blog qui peut initialement être un simple support d'archivage des recettes à destination d'un entourage proche. Cette situation entre en résonance avec le profil des blogueurs les moins actifs et les plus déconnectés de l'espace marchand, qui jouissent d'une audience très faible. Néanmoins, au fur et à mesure de la pratique, les blogueurs développent des liens nombreux avec leurs pairs qui constituent leur espace de référence. L'intégration à cette communauté va de pair avec le respect de certains principes d'interaction et pratiques de publication. L'augmentation de l'activité s'accompagne d'un accroissement de l'audience et consécutivement de la marchandisation du blog. Les partenariats les moins engageants (échanges produits) touchent ainsi un très grand nombre de blogueurs. L'établissement de partenariats plus poussés et plus nombreux suppose ensuite une activité de publication encore plus soutenue et une audience plus large. Les blogueurs se distinguent alors par un niveau de compétence déclaré et de complexité de la cuisine proposée plus élevé. Leur médiatisation montre que leur audience dépasse la seule blogosphère culinaire.

La capacité des blogueurs à émettre des évaluations qui soient entendues dépend donc en grande partie de leur activité et de leur audience, mais la « qualité » de l'audience compte aussi : selon qu'elle est composée unique- 
ment des pairs ou d'un public plus large, l'audience conduit à des partenariats de quantité et de qualité différente. Par ailleurs, en dépit du fait d'avoir en commun un fort investissement dans leur activité blogosphérique, les blogueurs les plus producteurs d'évaluations " commandées » sont plus différents entre eux que ne le sont les blogueurs dont l'audience est essentiellement limitée à la blogosphère.

Du point de vue de sa structure globale, il apparaît qu'à la différence des champs artistiques décrits par P. Bourdieu (1992), la blogosphère culinaire n'est pas organisée selon une opposition entre un pôle pur (créatif et non-commercial) et un pôle commercial (cherchant à plaire au plus grand nombre et visant le succès économique). Elle constitue plutôt un espace tripartite (forme en boomerang du nuage des individus dans l'ACM) : les blogueurs qui sont le plus éloignés de la commercialisation de leur blog sont aussi les moins actifs et les moins créatifs. Ils s'opposent aux blogueurs les plus créatifs (recettes plus élaborées, préoccupation esthétique, etc.) qui sont aussi les plus « commerciaux » (« l'élite »). L'opposition entre pureté et marchandisation doit ici être remise en question. 


\section{RÉFÉRENCES}

AGARWAL N., LIU H., \& TANG L. (2008), "Identifying the Influentials in Blogosphere", WSDM'08, Palo Alto, États-Unis.

ALLARD L. \& VANDENBERGHE F. (2003), « Express yourself! Les pages perso. Entre légitimation technopolitique de l'individualisme expressif et authenticité réflexive peer to peer $»$, Réseaux, $\mathrm{n}^{\circ} 117$, pp. 191-219.

BEAUDOUIN V. \& VELKOVSKA J. (1999), « Constitution d'un espace de communication sur Internet (forums, pages personnelles, courrier électronique...) », Réseaux, $\mathrm{n}^{\circ}$ 97, pp. 121-177.

BEAUVISAGE T., BEUSCART J.-S., CARDON V., MELLET K., \& TRESPEUCH M. (2013), « Notes et avis des consommateurs sur le Web. Les marchés à l'épreuve de l'évaluation profane », Réseaux, n 177, pp. 131-161.

BEUSCART J.-S. \& COURONNÉ T. (2009), « La distribution de la notoriété artistique en ligne. Une analyse quantitative de MySpace », Terrains \& Travaux, $\mathrm{n}^{\circ} 15$, pp. 147-170.

BOURDIEU P. (1989), La Noblesse d'État. Grandes écoles et esprit de corps, Paris : Minuit.

BOURDIEU P. (1992), Les Règles de l'art. Genèse et structure du champ littéraire, Paris : Seuil.

BROMBERGER C. (éd.) (1998), Passions ordinaires. Du match de football au concours de dictée, Paris : Bayard.

CARDON D. (2010), La Démocratie Internet. Promesses et limites, Paris : Seuil.

CARDON D. \& DELAUNAY-TÉTEREL H. (2006), « La production de soi comme technique relationnelle. Un essai de typologie des blogs par leurs publics », Réseaux, $\mathrm{n}^{\circ} 138$, pp. 5-71.

CARDON D., FOUETILlOU G., LERONDEAU C., \& PRIEUR C. (2011), « Esquisse de géographie de la blogosphère politique (2007-2009) », in F. GREFFET (éd.), Continuerlalutte.com. Les partis politiques sur le Web, Paris : Presses de Sciences Po, pp. 73-94.

CARDON D., FOUETILlOU G., \& ROTH C. (2011), “Two Paths of Glory. Structural Positions and Trajectories of Website within their Topical Territory", Intern. Conf. on Weblogs and Social Media (ICWSM), Barcelone, Espagne.

CHA M., KWAK H., RODRIGUEZ P., AHN Y.-Y., \& MOON S. (2007), "I Tube, You Tube, Everybody Tubes: analyzing the World's Largest User Generated Content Video System", IMC'07, San Diego, États-Unis. 
FLICHY P. (2010), Le Sacre de l'amateur. Sociologie des passions ordinaires à l'ère numérique, Paris : Seuil.

FLUCKIGER C. (2006), «La sociabilité juvénile instrumentée. L'appropriation des blogs dans un groupe de collégiens », Réseaux, n 138, pp. 109-138.

LE ROUX B. \& ROUANET H. (2010), Multiple Correspondence Analysis, Thousand Oaks: Sage Publications.

MELLET K. (2009), "Aux sources du marketing viral », Réseaux, n 157-158, pp. 267-292.

NAULIN S. (2010), « Qui prescrit aux prescripteurs ? Place et rôle des attachées de presse dans la construction de la prescription des critiques gastronomiques », Terrains \& Travaux, $\mathrm{n}^{\circ} 17$, pp. 181-196.

NAULIN S. (2012), Le Journalisme gastronomique. Sociologie d'un dispositif de médiation marchande, thèse de sociologie, Université Paris-Sorbonne, Paris.

PINCH T. \& KESLER F. (2011), "How Aunt Ammy Gets Her Free Lunch: A Study of the Top-Thousand Customer Reviewers at Amazon.com”, working paper, [en ligne], https://docs.google.com/viewer?a=v\&pid=sites\&srcid=ZnJlZWx1bmNoLm1lfHRlc 3R8Z3g6NDg0MjliMjM3OWQzMWRINw.

ROUQUETTE S. (2008), « Les blogs “extimes” : analyse sociologique de l'interactivité des blogs », tic\&société, 2, 1, [en ligne], http://ticetsociete.revues.org/412.

STOICA A., COURONNÉ T., \& BEUSCART J.-S. (2010), “To Be a Star Is Not Only Metaphoric: From Popularity to Social Linkage", Fourth International AAAI Conference on Weblogs and Social Media, AAAI Press. 


\section{Annexes}

Annexe 1. Exemple d'article de blog dédié à l'évaluation de produits (http://www.audreycuisine.fr/2008/09/les-produits-pataks/, consulté le 10 mars 2014)

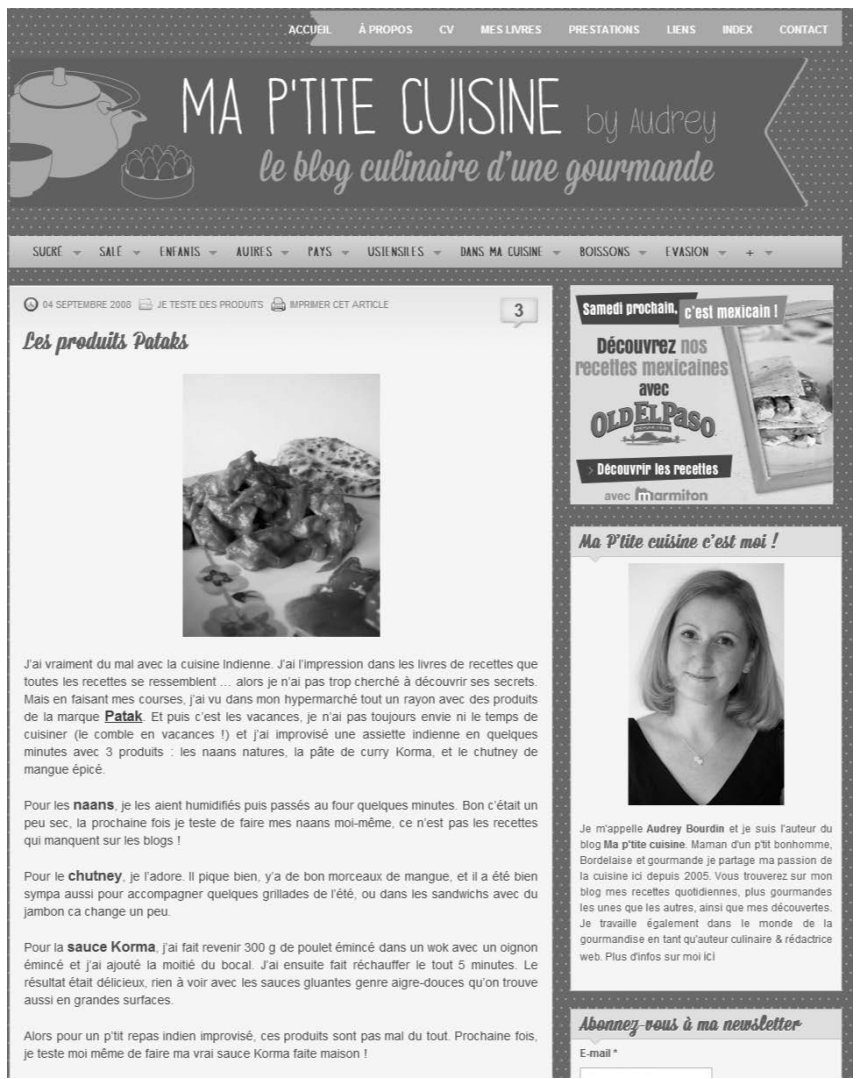


Annexe 2. Exemple de billet sponsorisé

(http://www.fashioncooking.fr/2012/06/recettes-autour-des-soupes-froides-alvalle/, consulté le 6 octobre 2012)

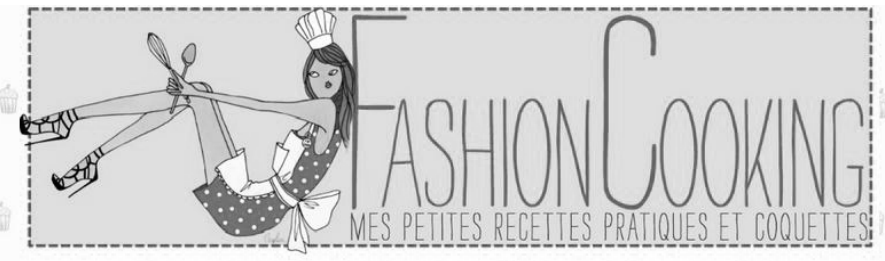

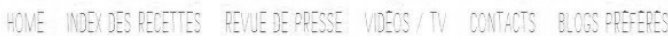
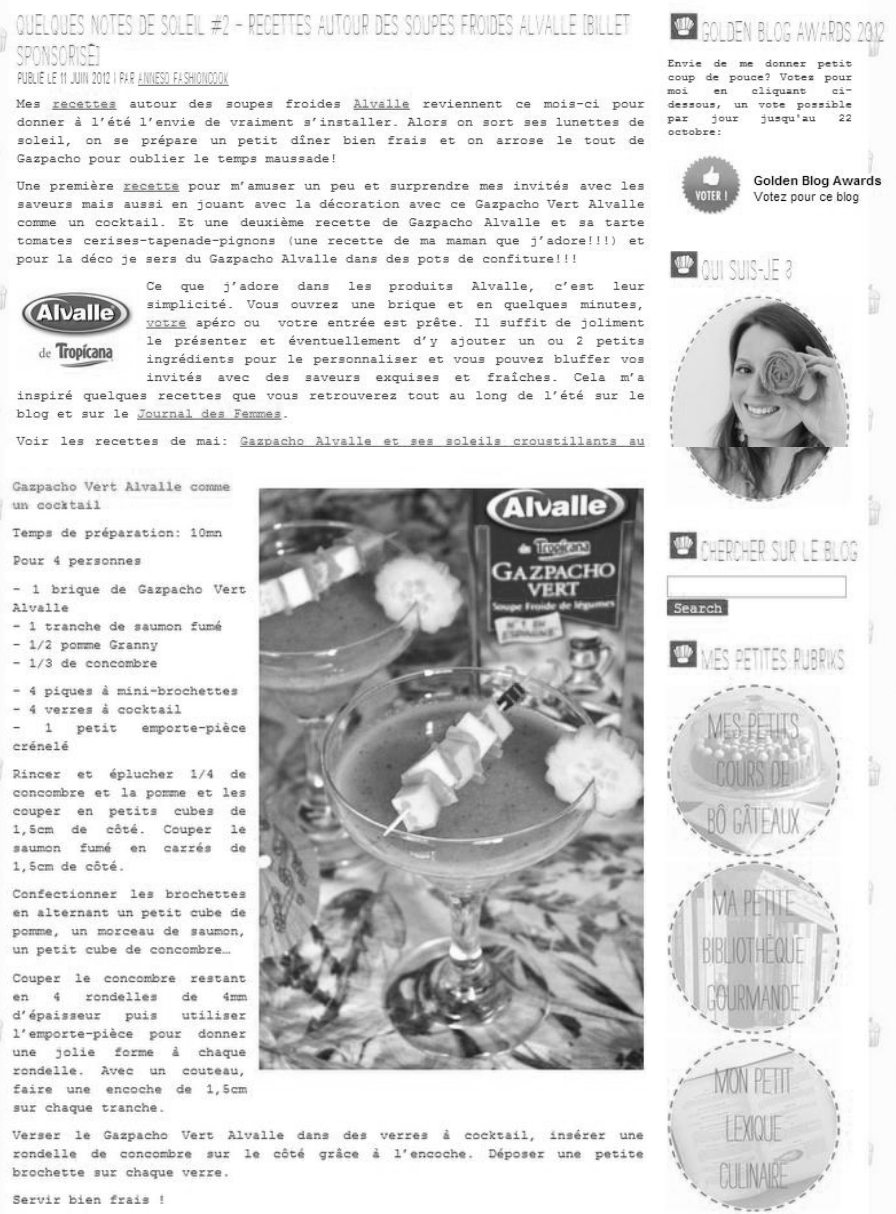
Annexe 3. Index des variables actives de l'ACM

\begin{tabular}{|l|}
\hline q3_DiffCuis \\
\hline Cuis_DeTousLesJours \\
Cuis_Créative \\
Cuis_NSP \\
\hline
\end{tabular}

\begin{tabular}{|l|}
\hline q5_FreqPubliBillets \\
\hline Freq_<1/Mois \\
Freq_1/Mois \\
Freq_Ts15Jours \\
Freq_1/Semn \\
Freq_2à6/Semn \\
Freq_1/Jour \\
\hline
\end{tabular}

\begin{tabular}{|l|}
\hline q6_FreqAvenir \\
\hline RalentirFreq \\
MaintienFreq \\
AugmFreq \\
\hline
\end{tabular}

\begin{tabular}{|l|}
\hline q7_NbCom \\
\hline <5Com \\
6à10Com \\
11à20Com \\
>20Com \\
\hline
\end{tabular}

\begin{tabular}{|l|}
\hline q8_NbVisiteurs \\
\hline Visit_0à10 \\
Visit_11à25 \\
Visit_26à100 \\
Visit_101à250 \\
Visit_251à1000 \\
Visit_>1000 \\
\hline
\end{tabular}

\begin{tabular}{|l|}
\hline q9_Stat \\
\hline Stat $<1 /$ mois \\
Stat 1 à $/$ mois \\
Stat $1 /$ semaine \\
Stat 2 à $6 /$ semaine \\
Stat $1 /$ jour \\
Stat $>1 /$ jour \\
\hline
\end{tabular}

\begin{tabular}{|l|}
\hline q10_NbHeuresBlog \\
\hline Blog 0 à $1 \mathrm{~h}$ \\
Blog 1 à $2 \mathrm{~h}$ \\
Blog 2 à $5 \mathrm{~h}$ \\
Blog 5 à $10 \mathrm{~h}$ \\
Blog $+10 \mathrm{~h}$ \\
\hline
\end{tabular}

\begin{tabular}{|l|}
\hline q14_MentionMedias \\
\hline MentionMedia \\
PasMentionPasProp \\
PasMentionRefus \\
\hline
\end{tabular}

\begin{tabular}{|l|}
\hline q29_ForumAvtCreaBlo \\
\hline ForumApresBlog \\
ForumAvtBlog \\
ForumNon \\
\hline
\end{tabular}

\begin{tabular}{|l|}
\hline q34_TypePartenariat \\
\hline Parten0 \\
PartenTestProduit \\
Parten+QueTestProdui \\
\hline
\end{tabular}

\begin{tabular}{|l|}
\hline q35_NbProdTest \\
\hline TestJamais \\
TestAucun2mois \\
Test 1 à 3 \\
Test 4 ou Plus \\
\hline
\end{tabular}

\begin{tabular}{|l|}
\hline q42_RapportBlog \\
\hline RapportRien \\
Rapport $<50$ \\
Rapport $>50$ \\
\hline
\end{tabular}

\begin{tabular}{|l|}
\hline q44_Professionnalisation \\
\hline DejaPro \\
VtResterAmat \\
VeutDevPro \\
\hline
\end{tabular}




\section{Annexe 4. Caractérisation de la partition des blogueurs par les modalités des classes ${ }^{37}$}

Classe 1/4 (Effectif : 205 - Pourcentage : 38,75)

\begin{tabular}{|c|c|c|c|}
\hline Libellés des variables & Modalités caractéristiques & $\begin{array}{l}\text { V a l e u r - } \\
\text { Test }\end{array}$ & Histogramme \\
\hline q34_recodTypePartenariat & PartenTestProduit & 13,81 & $* * * * * * * * * * * * * * * * * * * * * * * * * * * * * * * * * * * * * * * * *$ \\
\hline q42_recodRapportBlog & Rapport $<\mathbf{5 0}$ & 10,48 & $* * * * * * * * * * * * * * * * * * * * * * * * * * *$ \\
\hline q35_recodNbProd2Mois & Test 1 à 3 & 10,37 & $* * * * * * * * * * * * * * * * * * * * * * * * * * *$ \\
\hline q35_recodNbProd2Mois & TestAucun2mois & 8,37 & $* * * * * * * * * * * * * * * * * * * * * *$ \\
\hline q5_recod3FreqPubliBillets & 2a6ParSemaine & 6,06 & $* * * * * * * * * * * * * * * *$ \\
\hline q8_recod7NbVisiteurs & 251a1000Visit & 5,91 & $* * * * * * * * * * * * * * *$ \\
\hline q8_recod7NbVisiteurs & 101a250Visit & 5,21 & $* * * * * * * * * * * * *$ \\
\hline q10_recod5NbHeuresBlog & Blog 5 à $10 \mathrm{~h}$ & 5,11 & $* * * * * * * * * * * * *$ \\
\hline q29ForumAvtCreaBlo & ForumApresBlog & 4,45 & $* * * * * * * * * * *$ \\
\hline q7_recodNbComm & 11à20Comm & 2,98 & $* * * * * * * *$ \\
\hline q6_recod2FreqAvenir & MaintienFreq & 2,79 & $* * * * * * *$ \\
\hline q5_recod3FreqPubliBillets & UnParJour & 2,68 & $* * * * * * *$ \\
\hline q34_recodTypePartenariat & PartenAutreQueTestPr & 2,43 & $* * * * * *$ \\
\hline q9_recodStat & Stat $>1 /$ jour & 2,27 & $* * * * * *$ \\
\hline q7_recodNbComm & $>20$ Comm & 2,24 & $* * * * * *$ \\
\hline q9_recodStat & Stat1/semaine & 2,13 & $* * * * *$ \\
\hline
\end{tabular}

Classe 2/4 (Effectif : 64 - Pourcentage : 12,10)

\begin{tabular}{|l|l|l|l|}
\hline Libellés des variables & Modalités caractéristiques & Valeur-Test & Histogramme \\
\hline q42_recodRapportBlog & Rapport $>\mathbf{5 0}$ & 12,46 & $* * * * * * * * * * * * * * * * * * * * * * * * * * * * * * *$ \\
\hline q8_recod7NbVisiteurs & $>1000$ Visit & 12,23 & $* * * * * * * * * * * * * * * * * * * * * * * * * * * * * * *$ \\
\hline q34_recodTypePartenariat & PartenAutreQueTestPr & 6,72 & $* * * * * * * * * * * * * * * *$ \\
\hline q35_recodNbProd2Mois & Test 4 ou Plus & 6,72 & $* * * * * * * * * * * * * * * *$ \\
\hline q14CausePasMention & MentionMedia & 6,16 & $* * * * * * * * * * * * * * *$ \\
\hline q7_recodNbComm & $>20$ Comm & 5,65 & $* * * * * * * * * * * * * *$ \\
\hline q10_recod5NbHeuresBlog & Blog +10h & 5,54 & $* * * * * * * * * * * * * *$ \\
\hline q9_recodStat & Stat1/jour & 4,97 & $* * * * * * * * * * * * *$ \\
\hline q44_recodProfessionnalisation & DejaPro & 4,56 & $* * * * * * * * * * *$ \\
\hline q6_recod2FreqAvenir & MaintienFreq & 4,11 & $* * * * * * * * * * *$ \\
\hline q5_recod3FreqPubliBillets & UnParJour & 3,71 & $* * * * * * * * * *$ \\
\hline
\end{tabular}

37. En gras sont indiquées les modalités de succès commercial afférentes au pouvoir de prescription des blogueurs. 


\begin{tabular}{|l|l|l|l|}
\hline q5_recod3FreqPubliBillets & 2a6ParSemaine & 2,92 & $* * * * * * * *$ \\
\hline q7_recodNbComm & 11à20Comm & 2,15 & $* * * * * *$ \\
\hline q45Sexe & Homme & 1,98 & $* * * * *$ \\
\hline
\end{tabular}

Classe 3/4 (Effectif : 133 - Pourcentage : 25,14)

\begin{tabular}{|l|l|l|l|}
\hline Libellés des variables & Modalités caractéristiques & Valeur-Test & Histogramme \\
\hline q35_recodNbProd2Mois & TestJamais & 13,99 & $\begin{array}{l}* * * * * * * * * * * * * * * * * * * * * * * * * * * * * * * \\
* * *\end{array}$ \\
\hline q34_recodTypePartenariat & PartenAucun & 12,93 & $* * * * * * * * * * * * * * * * * * * * * * * * * * * * * * *$ \\
\hline q5_recod3FreqPubliBillets & UnParSemaine & 5,86 & $* * * * * * * * * * * * * *$ \\
\hline q42_recodRapportBlog & RapportRien & 5,64 & $* * * * * * * * * * * * *$ \\
\hline q8_recod7NbVisiteurs & 26a100Visit & 5,42 & $* * * * * * * * * * * *$ \\
\hline q7_recodNbComm & 6à10Comm & 3,81 & $* * * * * * * * * *$ \\
\hline q44_recodProfessionnalisation & VtResterAmat & 3,07 & $* * * * * * * *$ \\
\hline q6_recod2FreqAvenir & MaintienFreq & 2,73 & $* * * * * * *$ \\
\hline q54TailleCom & TailleComParis & 2,69 & $* * * * * * *$ \\
\hline q10_recod5NbHeuresBlog & Blog 2 à 5h & 2,35 & $* * * * * *$ \\
\hline q10_recod5NbHeuresBlog & Blog 1 à 2h & 2,13 & $* * * * *$ \\
\hline q14CausePasMention & PasMentionPasProp & 2,11 & $* * * * *$ \\
\hline
\end{tabular}

Classe 4/4 (Effectif : 127 - Pourcentage : 24,01)

\begin{tabular}{|l|l|l|l|}
\hline Libellés des variables & Modalités caractéristiques & Valeur-Test & Histogramme \\
\hline q5_recod3FreqPubliBillets & MoinsUnParMois & 13,21 & $* * * * * * * * * * * * * * * * * * * * * * * * * * * * * * * * *$ \\
\hline q10_recod5NbHeuresBlog & Blog 0 à $1 \mathrm{~h}$ & 10,12 & $* * * * * * * * * * * * * * * * * * * * * * * * *$ \\
\hline q7_recodNbComm & $<5$ Comm & 10,06 & $* * * * * * * * * * * * * * * * * * * * * * * * *$ \\
\hline q8_recod7NbVisiteurs & 0a10Visit & 10,03 & $* * * * * * * * * * * * * * * * * * * * * * * *$ \\
\hline q6_recod2FreqAvenir & AugmFreq & 9,65 & $* * * * * * * * * * * * * * * * * * * * * * *$ \\
\hline q42_recodRapportBlog & RapportRien & 9,14 & $* * * * * * * * * * * * * * * * * * * * * * *$ \\
\hline q9_recodStat & Stat $<1 /$ mois & 8,33 & $* * * * * * * * * * * * * * * * * * * *$ \\
\hline q35_recodNbProd2Mois & TestJamais & 7,91 & $* * * * * * * * * * * * * * * * * * *$ \\
\hline q34_recodTypePartenariat & PartenAucun & 7,90 & $* * * * * * * * * * * * * * * * * * *$ \\
\hline q8_recod7NbVisiteurs & $11 a 25 V i s i t$ & 6,85 & $* * * * * * * * * * * * * * * * *$ \\
\hline q5_recod3FreqPubliBillets & UnParMois & 6,11 & $* * * * * * * * * * * * * * *$ \\
\hline q9_recodStat & Stat1a3/mois & 4,47 & $* * * * * * * * * * * *$ \\
\hline q14CausePasMention & PasMentionPasProp & 4,40 & $* * * * * * * * * * *$ \\
\hline q29ForumAvtCreaBlo & ForumNon & 3,60 & $* * * * * * * * *$ \\
\hline q56_recod3Revenus_A & R $<2000$ & 2,16 & $* * * * * *$ \\
\hline
\end{tabular}

